\title{
To PEG or not to PEG
}

This article is based on a presentation at the RCP General Medicine

Course on 31

October 2001 by

Christopher Pennington $\mathrm{BSC}$ MD FRCP FRCP(Ed),

Consultant

Gastroenterologist, Honorary Professor of Clinical Nutrition,

Tayside University

Hospital Trust,

Ninewells Hospital and Medical

School, Dundee

Christopher Pennington

ABSTRACT - Patients with adequate intestinal function who are unable to eat may benefit from enteral tube feeding. Percutaneous endoscopic gastrostomy (PEG) is preferred when prolonged treatment is envisaged. PEG feeding will reduce morbidity and mortality in many such patients by reversing malnutrition. The increasing numbers of elderly patients with chronic diseases have resulted in an increased demand for PEG placement that has stretched resources. Many patients who are referred for PEGs are frail and the procedure is associated with complications. Careful management and support for the carers in the community are essential. Not all patients benefit from PEG feeding. The aim must be an improvement in the quality of life, not a prolongation of terminal disease.

Key words: benefits, community, CPD, disadvantages, duration, enteral tube feeding, ethics, indications, methods, organisation, prevalence

Patients who are unwilling or unable to eat or who have intestinal failure due to impaired digestion or absorption will starve. Starvation depletes tissue stores, and ultimately leads to impaired organ function and tissue structure. This will expose patients to the risk of disease, especially infection, and increase the length of hospital stay. Such patients require expert attention to nutritional management. For those patients who can or will eat, the improvement in the quality, type and availability of hospital and institutional food may be sufficient. Patients who cannot eat or absorb nutrients will require artificial nutrition support. When the intestinal function is adequate, enteral tube feeding is preferred to parenteral nutrition, not only because of cost and safety implications but because there are also benefits in relation to immune function and possibly the mucosal barrier function.

The increasing age of the population that is associated with degenerative disease has led to an escalating demand on medical technology that will outstrip current resources. The application of artificial nutrition support is one example that poses clinical and ethical dilemmas. This review will focus on some of the factors to be considered when determining patient selection for percutaneous endoscopic gastrostomy (PEG) feeding.

\section{Methods of enteral tube feeding}

Access to the intestinal tract may be via a nasal tube or by the percutaneous route, with delivery to the stomach or jejunum. Nasogastric tubes are employed for short-term delivery, usually up to four weeks. They are convenient to place, but frequently become displaced, especially in confused elderly patients. In this situation, they are associated with the risk of aspiration and inefficient nutrient delivery. They are also unsightly, although often surprisingly well tolerated in young patients. Nasojejunal tubes are used in subjects with gastric stasis to minimise the risk of reflux and aspiration, although the evidence to support the reduction in risk is lacking. Such an approach is more commonly used in intensive care, post-surgical patients and those with gastric stasis from any cause. Percutaneous access is usually by endoscopic techniques, with PEG or with the enteral end of the feeding tube advanced into the jejunum. Percutaneous endoscopic jejunostomy via the enteroscope is possible but difficult.

\section{Percutaneous gastrostomy}

Percutaneous gastrostomy can be achieved by three methods:

- endoscopic

- radiological

- surgical.

\section{Endoscopic}

Endoscopic placement may be by either the pushpull method described by Ponsky and Gauderer (Fig 1$)^{1}$ or the direct push technique ${ }^{2}$. Both approaches require:

- the patient to be fit enough to undergo endoscopy

- pathology that permits the passage of the endoscope into the stomach

- transillumination through the abdominal wall. 
Fig 1. Placement of the percutaneous endoscopic gastrostomy tube by the Ponsky-Gauderer method: (a) finger pressure on anterior abdominal wall noted by endoscopist; (b) needle and cannula inserted into stomach through anterior abdominal wall after local anaesthesia; guidewire threaded through cannula and grasped by endoscopic forceps or snare; (c) endoscope withdrawn with guidewire; guidewire attached to gastrostomy tube; guidewire and tube are pulled back through mouth, oesophagus and stomach to exit on anterior abdominal wall; (d) endoscope re-passed to confirm site of placement of retention device.

\section{Radiological}

Direct access under radiological control, percutaneous radiological gastrostomy (PRG), will circumvent some of these access problems. The stomach can be reliably identified, but a fine bore nasogastric tube is still needed for gastric distension.

\section{Surgical}

Laparoscopic surgical placement has also been employed. Prophylactic antibiotic treatment is recommended to avoid stomal contamination from the skin or luminal surface ${ }^{3}$.

\section{Replacement of percutaneous endoscopic gastrostomy tubes}

Once placed, PEG tubes are left in situ until signs of cracking appear, usually after 2-3 years - indeed, most tubes will survive longer than the patients in whom they are inserted. Damaged tubes need replacing; this can easily be done at the bedside, in the nursing home or at home, with a balloon gastrostomy. Younger and more mobile patients may prefer a flush button gastrostomy (Fig 2). This option should not be considered until the PEG tube has been in situ for six weeks, ensuring that the seal between the stomach and abdominal wall is intact and thus avoiding peritoneal soiling. Rapid replacement with a catheter, balloon gastrostomy or button should be undertaken - if tubes fall out, the gastrostomy closes. Replacement is also needed for tube occlusion that may occur when adequate protocols for flushing are not followed.

\section{Potential indications for percutaneous endoscopic gastrostomy}

The principal reasons for referral include:

- cerebrovascular disease

- chronic neurological disorders

- cancer

- gastrointestinal disease.

Fifteen per 1,000 of the population over the age of 75 develop a cerebrovascular problem, of whom two-thirds survive the first six months ${ }^{4}$. One-third suffer from dysphagia which will persist in a small proportion ${ }^{5}$. The dysphagia and/or nasogastric

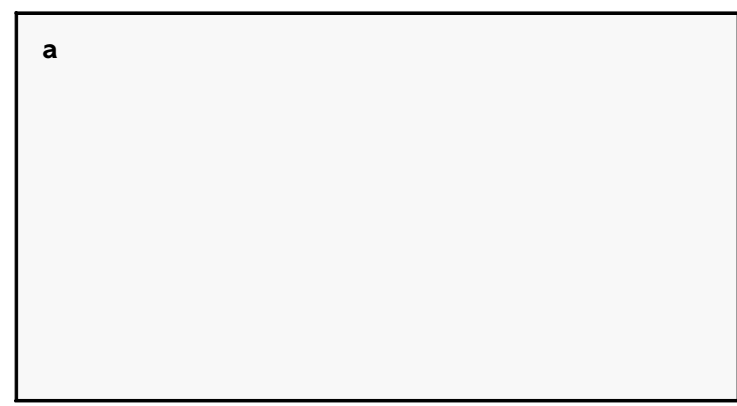

b
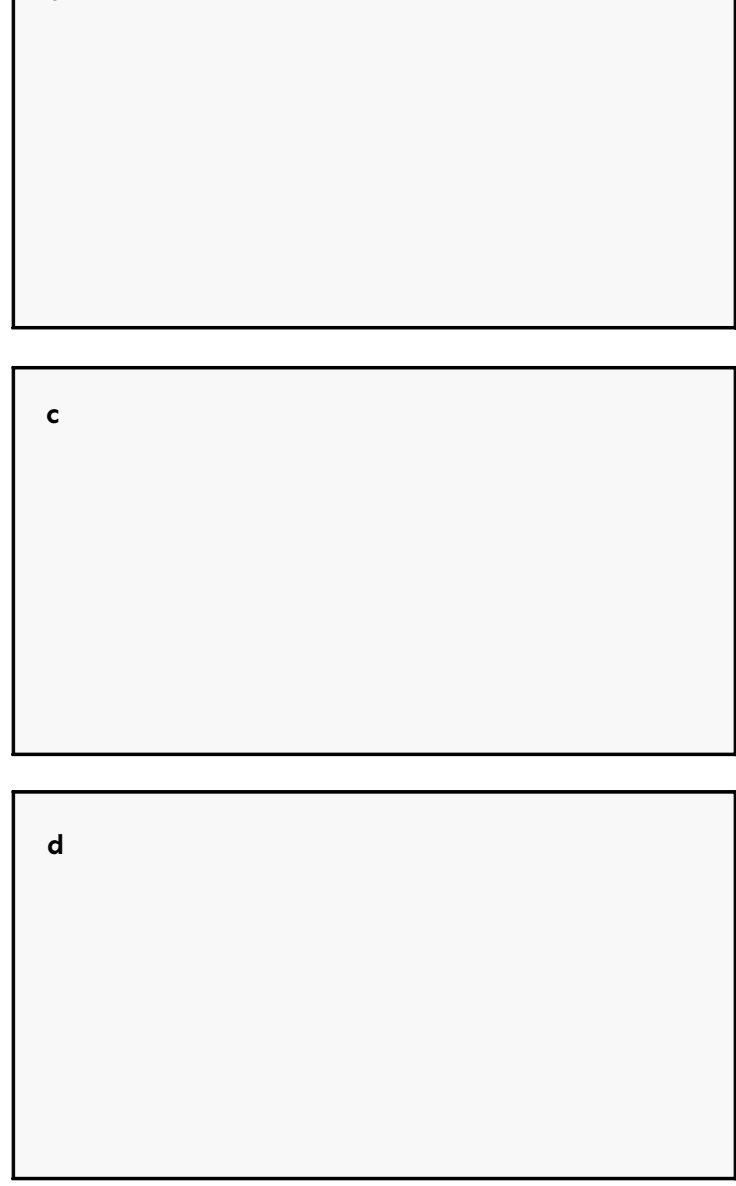

feeding may contribute to aspiration and chest infection, with premature death. Examples of chronic neurological diseases referred for PEG placement include:

- multiple sclerosis

- motor neurone disease

- Parkinson's disease, and

- dementia.

PEG placement is used to circumvent the dysphagia and anorexia in cancer patients. It is helpful for increasing the nutritional delivery in patients with cystic fibrosis and Crohn's disease. Conversely, PEG tubes may also be of value for the prevention of vomiting by de-functioning the stomach in 

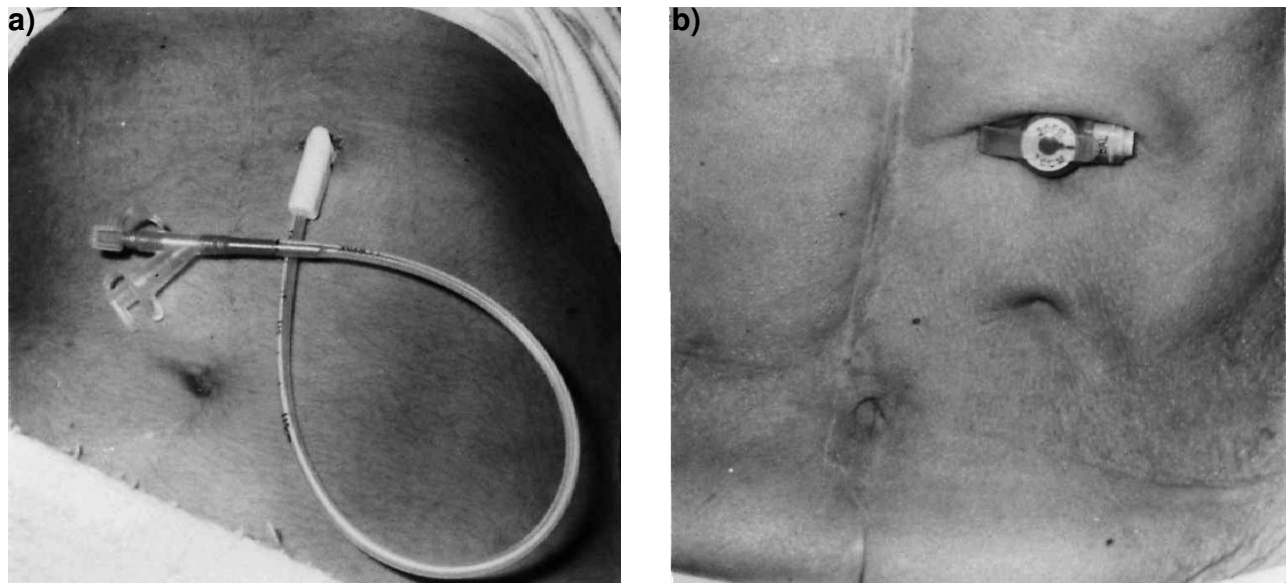

Fig 2. (a) A conventional percutaneous endoscopic gastrostomy tube; (b) a low profile gastrostomy 'button'. patients with untreatable obstruction through cancer, or advanced motility disorders such as hollow visceral myopathy.

\section{Contraindications to percutaneous endoscopic gastroscopy}

Contraindications for PEG placement include:

- patients who are unfit for endoscopy

- inability to pass the endoscope

- failure to transilluminate: this indicates that another organ may be between the abdominal wall and the stomach and the location of the stomach is unclear.

- (theoretically) patients with ascites: infection is a potential risk with ascites, especially through impaired apposition of the intraperitoneal gastric and abdominal surfaces.

\section{Percutaneous radiological gastrostomy (PRG)}

PRG may be an option in those patients who are unfit for gastrostomy and with failed transillumination when radiological imaging identifies the position of the stomach and other organs. It may also be considered when the instrument cannot be passed, especially due to neoplasia. There are a few case reports of metastasis to the exit site when traditional PEG methods are employed with oropharyngeal and oesophageal cancer ${ }^{6}$. This may reflect contamination as the PEG is pulled through the tumour using the Ponsky-Gauderer technique. Indeed, contamination of the endoscope itself using either endoscopic method could lead to contamination. PRG may be more suitable for such patients, but more follow-up information is needed in this area.

\section{Selection of patients}

The recognition of the potential value of PEG and the increasing numbers of patients with potential benefit have resulted in a rapid increase in the number of patients referred for placement and in the number of PEG tubes placed (Fig 3). Clearly, the importance of patient selection merits emphasis. Not all patients in whom there is an indication benefit from PEG tubes. In our unit in 2000, there were 130 placements of the 207 PEG referrals received. The corresponding projected numbers in 2001 are 254 referrals and 169 placements.

Patient selection is difficult, and raises clinical and ethical issues. The important consideration is the expected benefit to the patient.

\section{Prognosis in patients treated with percutaneous endoscopic gastrostomy feeding}

Many studies have demonstrated a mortality of about $30 \%$ during the 30 days after insertion. The British Association for Parenteral and Enteral Nutrition (BAPEN) continues to survey patients who receive home artificial nutrition support (the British Artificial Nutrition Survey (BANS) Register). Within the cohort of 16,247 patients, one year after starting home enteral tube feeding, mostly via PEG tubes, 50\% were still being fed in this way, $34 \%$ had died and $14 \%$ had returned to oral feeding. Only $1 \%$ were in hospital ${ }^{7}$.

\section{Dementia}

Clearly, the prognosis in those patients who suffer from prolonged inability to swallow will depend on diagnosis and age, in addition to concomitant disease and primary pathology. A study of survival in PEG feeding ${ }^{8}$ demonstrated a 54\% 30-day mortality in patients with dementia compared with $28 \%$ in all patients. By one year, $90 \%$ of the demented patients had died. The implication of this finding is that the inability to ingest food normally appears to be associated with the terminal phase of illness for patients with dementia, at least in the common forms of dementia such as Alzheimer's disease. In these circumstances, there is the problem of prolonging death, rather than providing benefit to the patients.

Other issues relating to dementia include the rare forms of the disease in which swallowing is lost at an earlier stage, and the temptation in many institutions to refer chronically disabled 


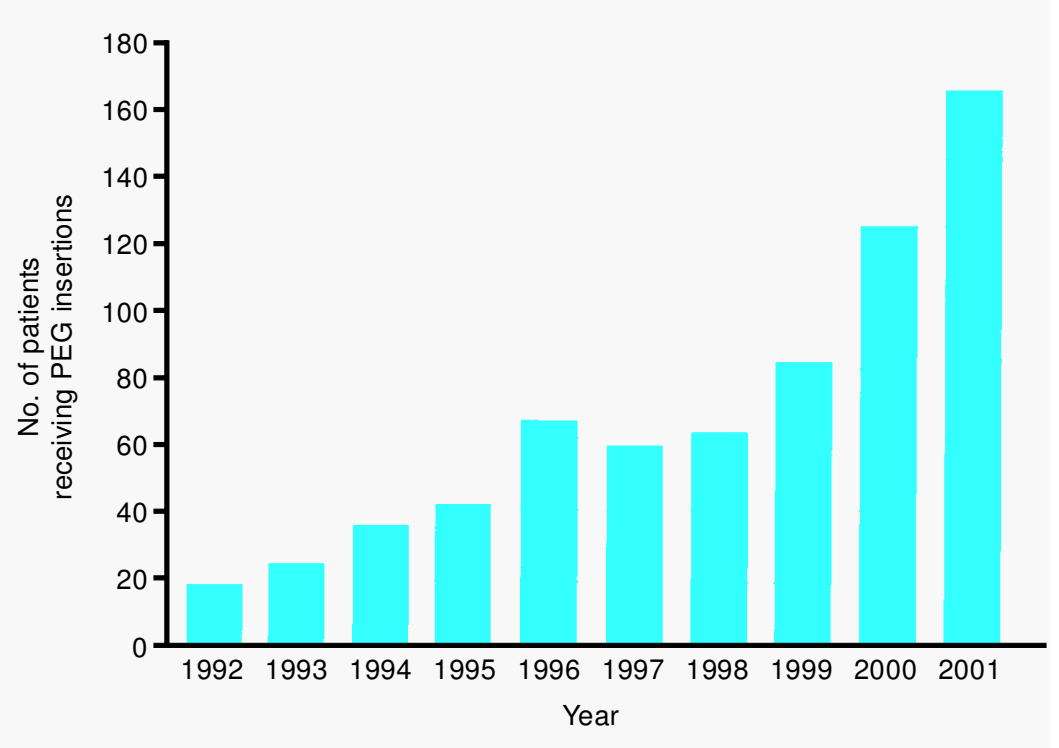

Fig 3. The number of patients undergoing percutaneous endoscopic gastrostomy (PEG) placement in Tayside University Hospital Trust 1992-2001. patients before PEG tubes are needed to relieve pressure on nursing time. This is not in the patients' interest. Careful assessment of their needs and abilities is important.

\section{Cerebrovascular disease}

Patients with cerebrovascular disease represent the largest single group referred for PEG insertion: there were 4,821 such patients in the BANS survey ${ }^{9}$. Excluding within-hospital mortality, there was $30 \%$ overall mortality one year after discharge - mainly due to the underlying disease. It was much lower in patients below the age of 64 and much greater in those over 75 . There were 553 and 2,777 patients within each respective group. Once PEG feeding is established, deaths occur through progression of the underlying disease, as also happens with the patients with chronic neurological disease. A patient's condition may remain static (eg cerebral trauma), progress slowly or deteriorate in a stepwise fashion. Delay or failure to provide this form of nutritional support can lead to a poor outcome due to significant complications associated with nutritional depletion or aspiration.

\section{Nutritional depletion}

The Irish hunger strikers demonstrated that fit young men die from starvation after about 70 days when they have lost 30-40\% of their body weight. However, starvation has a much more immediate impact in relation to organ function. The loss of muscle power and increased muscle fatiguability were evident in obese, otherwise fit subjects after two weeks without food ${ }^{10}$. This has implications for the frail and elderly in relation to mobility and falls in addition to respiratory function. Respiratory and immune impairment with nutritional depletion lead to infection.

Patients are judged to suffer from significant malnutrition with a body mass index (BMI) of 18.5, weight loss of $10 \%$ and starvation for 7-10 days. Body composition changes in the elderly so that for a given BMI their fat-free mass is lower than in a younger age group ${ }^{11}$. This suggests the need for greater sensitivity for the detection and recognition of existing or potential malnutrition. Indeed it is important to remember that the features of malnutrition, for example apathy, weakness and impaired immunity increase morbidity and reduce the quality of life. Furthermore, nutritional depletion takes a long time to recover - more so in the elderly ${ }^{12}$.

\section{Key Points}

Percutaneous endoscopic gastrostomy (PEG) is an important method of enteral tube feeding for patients who require longer-term artificial nutritional support to prevent or correct disease-related malnutrition

Patients who benefit from PEG include those with swallowing disorders, chronic neurological disease and cerebrovascular disease

Alternative methods such as percutaneous radiological endoscopy may be required in a minority of patients where PEG is not safe

PEG is not indicated in other patients, including some with Alzheimer's disease

The increase in the referral of patients for PEG has been considerable in most centres. Many such patients are elderly; this raises important issues of medical ethics in terms of medical complications and utilisation of resources

A PEG can be an advantage or a burden. The organisation of nutrition support within each hospital will have an important bearing on protocols, audit and management in the community in addition to the hospital setting 
PEG placement has the potential to prevent or reverse nutritional deficits, and thus improve fitness and quality of life for those patients unable to take sufficient oral nutrients. Through appropriate and timely intervention it may also prevent the problem of aspiration in patients with swallowing disorders. Nevertheless, depriving patients of food has an important impact on their social and eating experience.

\section{Ethical considerations}

The importance of ethical considerations was recognised in the time of Hippocrates. He extolled the principles of nonmalificence and beneficence. The principle of autonomy rather than paternalism and justice has now also been accepted ${ }^{13}$.

PEG placement is not without risk. Complications from endoscopy and the PEG procedure can be hazardous in this elderly population. They include peritoneal leak, haemorrhage and damage to abdominal viscera, and there is a small mortality. This is balanced against the benefits of nutrition and airway protection and set against the patient's prognosis.

Rabeneck and colleagues published ethical guidelines for PEG placement ${ }^{14}$. They advise against offering PEG to patients with the anorexia-cachexia syndrome because of an inability to utilise nutrients, and to those with permanent vegetative state (PVS) because such patients are unable to experience any quality of life. However, treatment may be needed to diagnose the anorexia-cachexia syndrome, and patients with muscle wasting through chronic neurological syndrome often benefit from PEG. The diagnosis of PVS can be made only after 6-12 months, depending on circumstances. Such patients will already be receiving PEG feeding; the issue here is discontinuing treatment. The authors recommend PEG treatment with dysphagia, but after discussion with patients with complications due to the potential for the loss of quality of life. They distinguish directive and non-directive counselling.

Paternalism used to be a tradition within the practice of medicine, but there has now been a move to autonomy. The patient must be informed, and it is then his or her decision whether or not to accept treatment. When patients are not competent to make this decision, it may be made as a result of discussion with relative(s) on their behalf by the consultant responsible for their care. Before such decisions are made, it is important to:

- assess the patient carefully

- fully discuss the problem with the relatives

- $\quad$ seek a living will, if such exists

- seek a second opinion if in doubt and, finally,

- seek the advice of the courts in rare circumstances ${ }^{15,16}$.

It is now recognised that financial resources within the NHS are limited. We have a responsibility to ensure that facilities are deployed where gain for the patient can be expected. The burden on patients and relatives should not be increased through useless PEG feeding.

\section{Organisation of percutaneous endoscopic gastrostomy}

The organisation of nutrition support within the hospital will have an important bearing both on the value of PEG to the patient and on the effective use of resource. Policies for patient selection should be in place, with guidelines for pre- and postinsertion care, including:

- the appropriateness of PEG in the patient

- the fitness for endoscopy

- protocols to include coagulation screen, antibiotic prophylaxis and consent from the patient (or relative if the patient is not competent).

Audit is an important barometer of patient selection and the quality of endoscopy. Patients who receive PEG are often elderly and frail. A procedure-related morbidity and mortality of less than $10 \%$ and $2 \%$, respectively, should be achieved ${ }^{17}$. Postinsertion care, training the patient or relative and follow-up into the community are essential. Support for the patient with the PEG at home, and a contact telephone number for advice about matters such as stomal leaking, blocked tubes and pump malfunction are an essential part of the service.

A PEG can be a great advantage or a burden. Improving the quality of life rather than prolonging death is very important. Nutrition support teams need to be well organised and follow the patient journey into the community. Working with the patient and the patient's carers is essential. The decision to place a PEG tube is never urgent, and must be taken after due consideration and discussion.

\section{References}

1 Gauderer MW, Ponsky JL, Izant RJ. Gastrostomy without laparotomy: a percutaneous endoscopic technique. J Paed Surg 1980;15:872-5.

2 Gorman RC, Morris JB. Minimally invasive access to the gastrointestinal tract. In: Rombeau JL, Rolandelli RH (eds). Clinical nutrition: enteral and tube feeding, 3rd edn. London: WB Saunders Co, 1990: Ch $11,174-5$

3 Sharma VK, Howden CW. Meta-analysis of randomized, controlled trials of antibiotic prophylaxis before percutaneous endoscopic gastrostomy. Am J Gastroenterol 2000;95:3133-6.

4 The Scottish Home and Health Department. The management of patients with stroke. Edinburgh: SHHD, 1993.

5 Barer DH. The natural history and functional consequences of dysphagia after hemispheric stroke. J Neurol Neurosurg Psychiatry 1989;52:236-41.

6 Douglas JG, Koh W, Laramore GE. Metastasis to a percutaneous gastrostomy site from head and neck cancer: radiobiologic considerations. Review. Head Neck 2000;22:826-30.

7 Elia ME, Russell CA, Stratton RJ, Holden CE, et al. In: Elia M, Russell $\mathrm{C}$, Stratton R (eds). Trends in artificial nutrition support in the UK during 1996-2000. Report by BANS. London: British Association for Parenteral and Enteral Nutrition, 2001.

8 Sanders DS, Carter MJ, D'Silva J, James G, et al. Survival analysis in percutaneous endoscopic gastrostomy feeding: a worse outcome in patients with dementia. Am J Gastroenterol 2000;95:1472-5.

9 Elia M, Stratton RJ, Holden C, Meadows N, et al. Home enteral tube feeding following cerebrovascular accident. Clin Nutr 2001;20:27-30.

10 Jeejeebhoy KN. Bulk or bounce - the object of nutritional support. Review. Rhoads Lecture 1988. J Parenter Enteral Nutr1988;12:539-49. 
11 Hébuterene X, Bermon S, Schneider SM. Ageing and muscle: the effects of malnutrition, re-nutrition, and physical exercise. Curr Opin Clin Nutr Metabol Care 2001;4:295-300.

12 Hébuterene X, Péroux J, Schneider S, Rampal P. Effects of refeeding by cyclic enteral nutrition on body composition: comparative study of elderly and younger patients. Clin Nutr 1997;16:283-9.

13 MacFie J. Ethics and nutritional support therapy: a clinician's view. Clin Nutr 2001;20(Suppl 1):87-99.

14 Rabeneck L, McCullough LB, Wray NP. Ethically justified, clinically comprehensive guidelines for percutaneous endoscopic gastrostomy tube placement. Lancet 1997;349:496-8.

15 Lennard-Jones JE. Ethical and legal aspects of clinical hydration and nutritional support. London: The British Association for Parenteral and Enteral Nutrition, 1999.

16 Smith S. The role of the court in ethical decision making. Clin Med 2001;1: 371-3.

17 Payne-James J. Enteral nutrition: tubes and techniques of delivery. In: Payne-James J, Grimble G, Silk DB (eds). Artificial nutrition support, 2nd edn. London: Greenwich Medical Media, 2001: Ch 17, 290-1. 